\title{
Analyzing the Route Cache Timeout Parameter of DSR Protocol in Mobile Ad Hoc Networks
}

\author{
Barış Özyurt ${ }^{*}$, İbrahim Alper Doğru², Muhammet Ali Akcayol ${ }^{3}$ \\ ${ }^{1}$ Dept. of Computer Science, Gazi University, Ins. of Information, Tunus Cad. Çankaya, Ankara, Turkey. \\ 2 Dept. of Computer Eng., Gazi University, Faculty of Technology, Teknikokullar, Ankara, Turkey. \\ ${ }^{3}$ Dept. of Computer Eng., Gazi University, Faculty of Engineering, Maltepe, Ankara, Turkey. \\ * Corresponding author. Tel.: 0-545-9044349; email: b.ozyurt@gmail.com \\ Manuscript submitted January 28, 2016; accepted August 1, 2016. \\ doi: 10.17706/ijcce.2017.6.1.40-48
}

\begin{abstract}
Routing is an important issue in Mobile ad hoc Networks (MANET). Several routing protocols have been developed in MANET so far. Dynamic Source Routing (DSR) is one of the most commonly used routing protocols in mobile ad hoc networks. DSR is a reactive routing protocol which means that when a node wants to send a packet, it has to make a route discovery to the destination node. When the mobility increases in the network, making route discovery and sending packet to the destination node becomes much more challenging. Increased mobility reduces the performance of all MANET routing protocols. DSR is no exception; increased mobility reduces the performance of DSR protocol and decreases its throughput. In this paper, we present our analysis about the effects of the "Route Cache Timeout" parameter on the performance of DSR protocol.
\end{abstract}

Key words: Mobile ad hoc networks, routing, DSR protocol, route cache timeout.

\section{Introduction}

Mobile ad hoc Networks (MANET) are self-organizing and self-configuring networks. They neither have an infrastructure nor require an administration. Each node in the network also acts as a router. They differ from traditional networks by having no infrastructure, nodes being mobile and communication being carried out wirelessly by electro-magnetic waves.

Because of these differences, routing protocols used in traditional networks cannot be used in mobile ad hoc networks, so special routing protocols have been developed for these networks. MANET routing protocols are generally treated in two categories: Proactive routing protocols and reactive routing protocols. In proactive routing protocols, each node holds the network topology and periodically sends "hello" messages in order to keep their network topology information up-to-date. Destination-Sequenced Distance Vector (DSDV) is the most popular proactive routing protocol [1].

In reactive routing protocols, the nodes do not hold the network topology. When a node wants to send a packet, firstly it makes "route discovery" to find a route to the destination node. Dynamic Source Routing (DSR) [2] and Ad hoc On-demand Distance Vector (AODV) [3] are the most popular reactive routing protocols.

As stated above, DSR is a popular MANET routing protocol proposed by D. Johnson and D. Maltz in 1996. Since that time, DSR has been being used in many ad hoc network implementations. Like all other routing protocols, DSR has some parameters to be tuned. Before using DSR protocol in a real life ad hoc network 
implementation, to get optimum performance and throughput, these parameters should be tuned properly.

Route Cache Timeout is one of the parameters of DSR protocol. But, to be able to tune this parameter properly, how it affects the performance in ad hoc networks with different characteristics should be properly comprehended. In this paper, we present our research about the effects of the "Route Cache Timeout" parameter on the performance of DSR protocol. We carried out our research using the Omnet++ network simulator.

\section{Related Work}

Lots of work has been done so far in order to analyze the performance and throughput of MANET routing protocols. Also in the literature, there are pretty number of papers published about the performance analysis of MANET routing protocols and about works done to improve throughput of these protocols.

S. Mohapatra and P. Kanungo made an analysis on the performance of AODV, DSR, OLSR and DSDV routing protocols using NS-2 network simulator [4]. In their work, they figured out that in small size networks, DSR protocol performs best in the Packet Delivery Rate (PDR) performance measure. But they found that, in large size networks, OLSR protocol performs best in PDR performance measure. Asma Tuteja et al. compared DSR, AODV and DSDV protocols using NS-2 network simulator [5]. In their work, they found that DSDV protocol's throughput is the lowest and its routing load is very high. They figured out that in some situations AODV performed better than DSR, but in the overall, DSR outperformed AODV in the end-to-end-delay performance measure.

M. Tamilarasi et al. made a work on DSR to eliminate the stale routes in the route cache [6]. They put forward a heuristic that "Routes having more hop counts are more vulnerable to get stale" and proposed an algorithm implementing a varying Route Cache Timeout mechanism according to the hop count. Nikhil I. Panchal et al. proposed some techniques for route cache optimization [7]. Their techniques include route cache validation, path enhancement through periodic searches to find shorter and more reliable path than that are present in the cache, path pruning by detecting low quality paths by using a heuristic algorithm. Hu and Johnson made a detailed analysis on route caches of the DSR protocol [8]. In their work, they analyzed cache structure, cache capacity and cache timeout using the NS-2 network simulator.

A. Kumar et al. made a comparative analysis of DSR, AODV, DSDV and CBRP manet routing protocols using the NS-2 simulator [9]. Their observations from the simulation results are as follows: When the mobility increases all routing protocols' throughput and average delay performance decreases except for AODV. Its throughput and average delay performance increases with increasing mobility. According to the packet delivery ratio performance metric, CBRP protocol outperforms the other three protocols.

A. O. Abu Salem et al. made analysis about the route cache sizes in DSR protocol using the NS-2 simulator [10]. In the simulation scenarios, they used ad hoc networks having relatively high mobility. From the simulation results, they observed that route cache size significantly affects the DSR ptotocol's performance. In their simulation scenarios, they observed that optimum performance is obtained when primary route cache size is between 5 and 10 and secondary route cache size is between 10 and 20 .

Besides the researches about the performance analysis of DSR and other routing protocols, some other researches have been made to enhance the performance of DSR protocol. Bin Xiao et al. proposed the "route recovery" method for DSR protocol which will be used in the case of a broken link [11]. In Fig. 1, we see a route $<\mathrm{a}, \mathrm{b}, \mathrm{c}, \mathrm{e}, \mathrm{f}>$ from node $\mathrm{a}$ to node $\mathrm{b}$. The $\mathrm{b}-\mathrm{c}$ link gets broken, because node $\mathrm{c}$ moves away. In this case, when a packet comes to node b, a Route Error (RERR) packet is sent to node a, which informs it about the broken link. In the proposed "route maintenance" method, the RERR packet is not immediately sent to source node. Instead, an alternative route is searched for the unreachable node (node $c$ in this case) in the route cache of node $b$. 


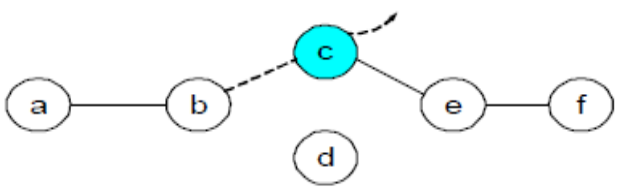

Fig. 1. Route maintenance example.

If an alternative route can be found, then that route is used for the communication. If an alternative route cannot be found, then an alternative route is searched in route caches of all nodes which are on the way to the source node. If an alternative route cannot be found in route caches of all these nodes, finally the RERR packet is sent to the source node. Bin Xiao proved that this method enhances the throughput of DSR protocol by making tests in simulation environment.

Zaiba Ishrat et al. proposed a more effective method for route selection in DSR protocol [12]. In DSR protocol, if there is more than one route from source to destination, the route having minimum hop count is preferred. Zaiba et al. proposed the "path ranking" technique. In this technique, each node in the network is ranked according to some criteria and the rank of each node is updated periodically. The rank of a route is the sum of all nodes' ranks on the route. When there is more than one route from source to destination, the route having the highest rank is chosen. For instance, when there is congestion at a node, its rank is lowered. By this way, the nodes having congestion is less preferred.

\section{DSR Protocol}

Dynamic Source Routing is a reactive MANET routing protocol [2]. In DSR, the nodes do not keep the network topology. When a node want to send a packet, first it makes a route discovery to the destination node, then it sends the packet through the route it has discovered.

\subsection{Route Discovery}

In DSR, route discovery is made with Route Request (RREQ) packets [2]. Source node emits a RREQ packet. The RREQ packet is delivered through the nodes in the network until it reaches the destination node. Each intermediate node delivering the RREQ packet, adds itself to the "Route Info" part of the RREQ packet. The destination node receiving the RREQ prepares the Route Reply (RREP) packet and sends it to the source node through the route RREQ packet came. When the RREP packet arrives at the source node, it contains the route to the destination node. This mechanism is depicted in Fig. 2 [12]. In the figure, the source node 1 makes a route discovery to the destination node 8.

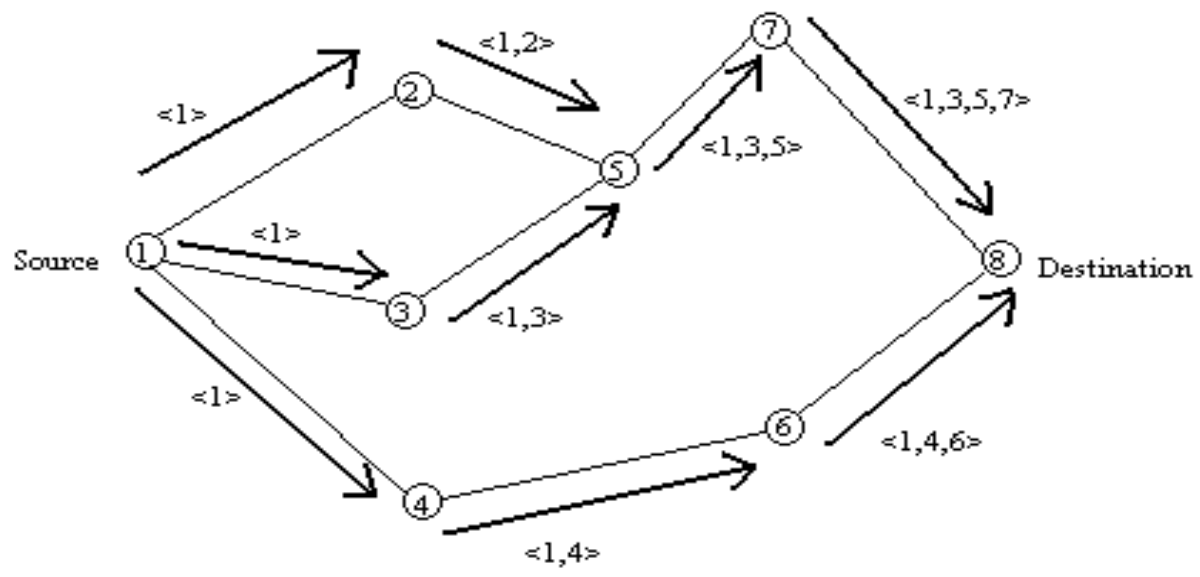

Fig. 2. The route request (RREQ) working mechanism. 


\subsection{Route Cache Mechanism}

Route Cache mechanism is used to enhance the performance of DSR protocol. When the RREQ and RREP packets propagate in the network, the nodes collect information from the "route info" data of these packets.

Nodes have access to a large amount of routing information. For instance, using a single request-reply cycle, the source node can learn routes to each intermediate node on the route. Each intermediate node can also learn routes to every other node on the route [13].

When a node receives a RREQ, first it checks its route cache. If the node can find a route to the destination node, it prepares the RREP packet and sends it to the source node. But here, there is a problem. Because of the mobility in the network, the route caches may become outdated in a few minutes later. In DSR, for his purpose the route caches have a "timeout" parameter. While configuring the DSR protocol, a timeout value (in seconds) is set to the "Route Cache Timeout" parameter. When a route is added to the route cache of a node, it is valid for "Route Cache Timeout" seconds and it expires after "Route Cache Timeout" seconds pass.

\section{Route Cache Timeout Analysis}

Route Cache Timeout is one of the important parameters of DSR protocol affecting the throughput of the protocol. In this paper, we present our research about the effects of the Route Cache Timeout parameter on the performance of DSR protocol. It's important to see the effect of Route Cache Timeout on ad hoc networks with different characteristics. For example, ad hoc networks with different number of nodes and scales; also ad hoc networks having different levels of mobility have been used in this work. We carried out our research using the Omnet++ network simulator.

\subsection{Simulation Environments}

We wanted to test the effects of the Route Cache Timeout parameter in networks with high mobility and low mobility. Also, for the sake of scalability of our analysis, ad hoc networks having different number of nodes and having different scales have been used.

To meet the above ad hoc network variety, four different simulation environments have been created. High mobility ad hoc network having 25 nodes, high mobility ad hoc network having 50 nodes, low mobility ad hoc network having 25 nodes and low mobility ad hoc network having 50 nodes. The properties of these simulation environments are shown in Table 1 and Table 2.

Table 1. Properties of High Mobility Simulation Environments

\begin{tabular}{|c|c|c|}
\hline & $\begin{array}{l}\text { High mobility Ad Hoc network having } \\
25 \text { nodes }\end{array}$ & $\begin{array}{l}\text { High mobility Ad Hoc network having } \\
50 \text { nodes }\end{array}$ \\
\hline Region size & $1000 \mathrm{~m} \times 800 \mathrm{~m}$ & $1400 \mathrm{~m} \times 1150 \mathrm{~m}$ \\
\hline Number of nodes & 25 & 50 \\
\hline Number of fixed and mobile nodes & 6 nodes fixed 19 nodes mobile & 12 nodes fixed 38 nodes mobile \\
\hline UDP Packet load of network & $\begin{array}{l}11 \text { nodes are sending UDP packets } \\
\text { periodically every } 2 \text { seconds }\end{array}$ & $\begin{array}{l}11 \text { nodes are sending UDP packets } \\
\text { periodically every } 2 \text { seconds }\end{array}$ \\
\hline Mobile nodes moving speed & $4 \mathrm{~m} / \mathrm{s}$ & $4 \mathrm{~m} / \mathrm{s}$ \\
\hline $\begin{array}{l}\text { Change in mobile nodes' moving } \\
\text { direction }\end{array}$ & By $30^{\circ}$ every 5 seconds & By $30^{\circ}$ every 5 seconds \\
\hline
\end{tabular}

As described above, for the scalability of our analysis both high and low mobility ad hoc networks have been constructed with 25 and 50 nodes. An important point to mention here is that we wanted these ad hoc networks having 25 nodes and 50 nodes to have similar network characteristics. 
Table 2. Properties of Low Mobility Simulation Environments

\begin{tabular}{|c|c|c|}
\hline & $\begin{array}{l}\text { Low mobility Ad Hoc Network having } \\
25 \text { nodes }\end{array}$ & $\begin{array}{l}\text { Low mobility Ad Hoc Network having } \\
50 \text { nodes }\end{array}$ \\
\hline Region size & $1000 \mathrm{~m} \times 800 \mathrm{~m}$ & $1400 \mathrm{~m} \times 1150 \mathrm{~m}$ \\
\hline Number of nodes & 25 & 50 \\
\hline Number of fixed and mobile nodes & 16 nodes fixed 9 nodes mobile & 32 nodes fixed 18 nodes mobile \\
\hline UDP Packet load of network & $\begin{array}{l}11 \text { nodes are sending UDP packets } \\
\text { periodically every } 2 \text { seconds }\end{array}$ & $\begin{array}{l}11 \text { nodes are sending UDP packets } \\
\text { periodically every } 2 \text { seconds }\end{array}$ \\
\hline Mobile nodes moving speed & $1 \mathrm{~m} / \mathrm{s}$ & $1 \mathrm{~m} / \mathrm{s}$ \\
\hline $\begin{array}{l}\text { Change in mobile nodes' moving } \\
\text { direction }\end{array}$ & By $30^{\circ}$ every 5 seconds & By $30^{\circ}$ every 5 seconds \\
\hline
\end{tabular}

For example ad hoc networks with 25 nodes have a simulation area of $1000 \mathrm{~m} \times 800 \mathrm{~m}=800.000 \mathrm{~m} 2$. Whereas ad hoc networks with 50 nodes have simulation area of $1400 \mathrm{~m} \times 1150 \mathrm{~m}=1.601 .000 \mathrm{~m} 2$ which is nearly two times the 25 nodes simulation area. By this way the density of nodes in both 25 and 50 nodes networks are nearly the same. Similarly, the ratio of mobile and fixed nodes in 25 nodes networks is same with 50 nodes networks. So the mobility levels are also the same.

To see the effects of Route Cache Timeout parameter on the performance of DSR protocol, each of the above four simulation scenarios have been run with 150s, 120s, 90s, 60s, 30s and 10s Route Cache Timeout values. The "Packet Delivery Rate (PDR)" of each run has been collected. PDR is the percentage of successfully delivered packets to the destination nodes. PDR is an important, may be the most important performance metric for ad hoc networks.

\subsection{Simulation Results}

Four simulation scenarios (high mobility 25 nodes, high mobility 50 nodes, low mobility 25 nodes, low mobility 50 nodes) and six different Route Cache Timeout values (150s, 120s, 90s, 60s, 30s, 10s), totally 24 simulations have been run in our analysis. Each simulation scenario has been run for 20 minutes. In each simulation scenario, 11 nodes are periodically sending UDP packets every 2 seconds, so the number of UDP packets sent in each scenario is the same. 11 nodes totally have sent 6454 UDP packets in each simulation scenario.

We will analyze the simulation results in two sub-titles: High Mobility Ad Hoc Network Simulation Results and Low Mobility Ad Hoc Network Simulation Results.

\subsubsection{High mobility ad hoc network simulation results}

Table 3. High Mobility Ad Hoc Network Simulation Results

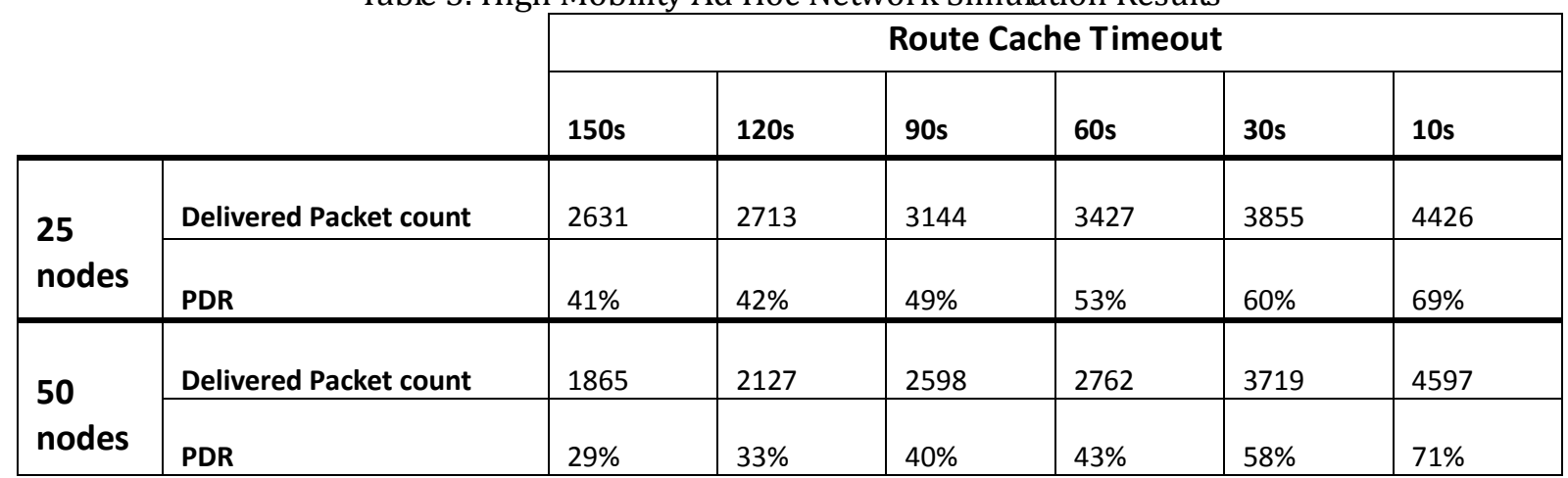

Total numbers of UDP packets successfully delivered and corresponding Packet Delivery Rate (PDR) values in high mobility ad hoc network are shown in Table 3. Results for 150s, 120s, 90s, 60s, 30s and 10s 
Route Cache Timeout values with 25 nodes and 50 nodes are shown separately. To better visually observe the results, they are graphically depicted in Fig. 3.

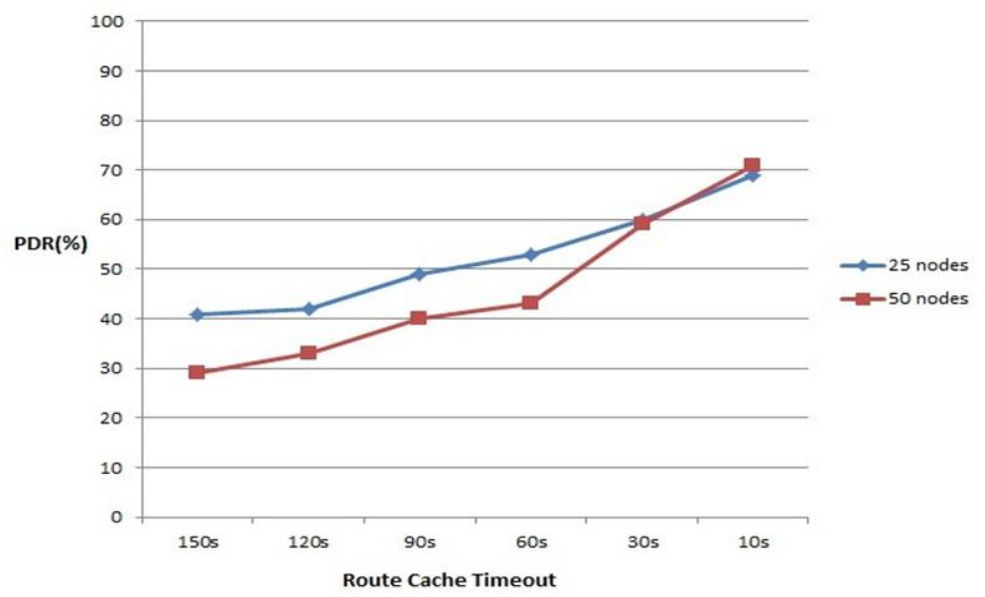

Fig. 3. High mobility ad hoc network simulation results.

\subsubsection{Low mobility ad hoc network simulation results}

Total numbers of UDP packets successfully delivered and corresponding Packet Delivery Rate (PDR) values in low mobility ad hoc network are shown in Table 4. Results for 150s, 120s, 90s, 60s, 30s and 10s Route Cache Timeout values with 25 nodes and 50 nodes are separately shown in Table 4 . To better visually observe the results, they are graphically depicted in Fig. 4.

Table 4. Low Mobility Ad Hoc Network Simulation Results

\begin{tabular}{|c|c|c|c|c|c|c|c|}
\hline & & \multicolumn{6}{|c|}{$\begin{array}{ll}\text { Route Cache Timeout } \\
\end{array}$} \\
\hline & & 150s & 120s & 90s & $60 \mathrm{~s}$ & 30s & $10 \mathrm{~s}$ \\
\hline \multirow{2}{*}{$\begin{array}{l}25 \\
\text { nodes }\end{array}$} & Delivered Packet count & 4930 & 5091 & 5379 & 5716 & 5611 & 5407 \\
\hline & PDR & $76 \%$ & $79 \%$ & $83 \%$ & $89 \%$ & $87 \%$ & $84 \%$ \\
\hline \multirow{2}{*}{$\begin{array}{l}50 \\
\text { nodes }\end{array}$} & Delivered Packet count & 4530 & 4535 & 5144 & 5537 & 5157 & 5085 \\
\hline & PDR & $70 \%$ & $70 \%$ & $80 \%$ & $86 \%$ & $80 \%$ & $79 \%$ \\
\hline
\end{tabular}

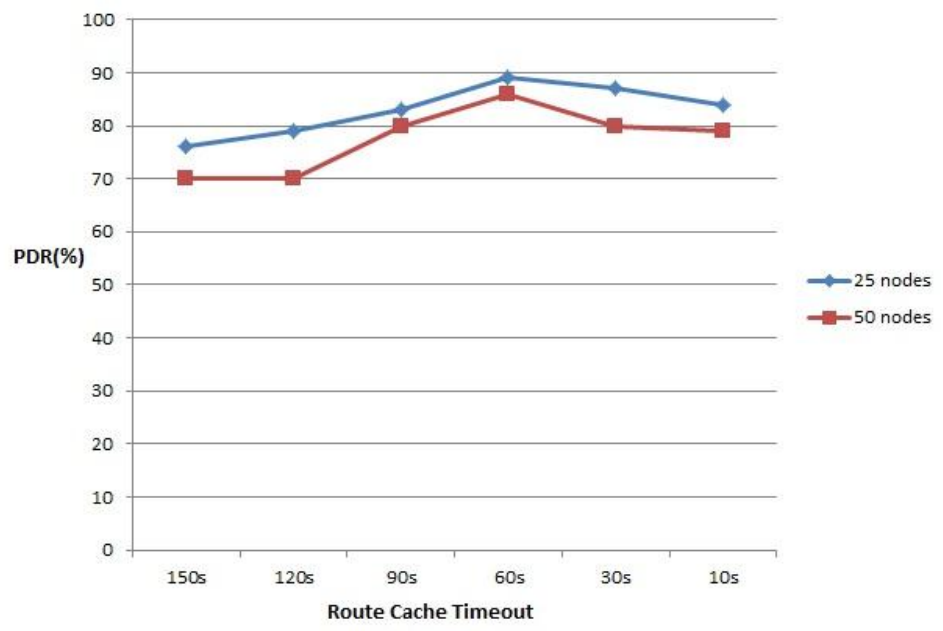

Fig. 4. Low mobility ad hoc network simulation results. 


\subsubsection{Comparing and analyzing the simulation results}

The first deduction we can make from the simulation results is that the Route Cache Timeout parameter has a significant effect on the performance of DSR protocol. This effect is much more significant in high mobility network than in low mobility network. In high mobility network with 50 nodes, reducing Route Cache Timeout from 150s to 10s enhances the PDR from $29 \%$ to $71 \%$. This is a drastic improvement in the performance. It can clearly be concluded that Route Cache Timeout is a very important parameter of the DSR protocol.

Looking at the results of high mobility simulation with 25 nodes, it is observed that 150s Route Cache Timeout yields the worst PDR output for the DSR protocol. Each incremental reduction in the Route Cache Timeout enhances the performance of the DSR protocol and finally 10s yields the best PDR output.

Looking at the results of high mobility simulation with 50 nodes, a similar picture is observed. 150s Route Cache Timeout yields the worst PDR value and incrementally reducing the Route Cache Timeout enhances the PDR performance of the DSR protocol. But the PDR values are slightly worse than 25 nodes. This is an expected situation because in the ad hoc network with 50 nodes, there are more hops between source and destination nodes with respect to 25 nodes. More hops mean more packet losses. These results are compliant with the results in [8]. In [8], $\mathrm{Hu}$ and Johnson used a high mobility (they say dynamic) simulation scenario with 40s, 20s, 10s, 2s and 1s Route Cache Timeout values. 40s yielded the worst PDR value and smaller values for Route Cache Timeout yielded better PDR values.

Analyzing the high mobility simulations' results gives the feeling that reducing the Route Cache Timeout value always enhances the performance of the DSR protocol. So, to enhance the performance of the DSR protocol, quite small values should be set to the Route Cache Timeout parameter.

On the other hand, looking at the low mobility ad hoc network simulation results, it is observed that the behavior of PDR according to Route Cache Timeout parameter is quite different than its behavior in high mobility network. Incrementally reducing the Route Cache Timeout from 150s to 60s enhances the PDR value but after 60 s the behavior changes. Reducing the Route Cache Timeout from $60 \mathrm{~s}$ to $30 \mathrm{~s}$ and then from 30s to 10s worsens the PDR output. 60s Route Cache Timeout value gives the best PDR output. This behavior is nearly the same in both networks having 25 nodes and 50 nodes.

In low mobility simulation results, it's observed that reducing the Route Cache Timeout parameter enhances the PDR performance of the DSR protocol until some point. After that point, continuing to reduce it, does not enhance the performance, contrarily worsens the performance of the DSR protocol.

Finally we can conclude from both high mobility and low mobility ad hoc network simulation results that the effect of changing the Route Cache Timeout parameter on the performance and throughput of DSR protocol depends on the mobility level of the network. So to maximize the performance of DSR protocol, the mobility of the ad hoc network should be analyzed and an optimum Route Cache Timeout value should be used according to the mobility level.

The deductions we made from simulation results is true for simulation scenarios with both 25 nodes and 50 nodes. This verifies the scalability of our analysis. Simulation results revealed that different scale ad hoc networks having similar characteristics present similar behaviors.

\section{Conclusion}

In this paper, "Route Cache Timeout" parameter of the DSR protocol is analyzed using the Omnet++ network simulator. This parameter is analyzed both in high mobility and low mobility ad hoc networks. High mobility and low mobility simulation environments were created in the Omnet++ simulator and the simulation scenarios were run with 150s, 90s, 60s, 30s and 10s Route Cache Timeout values. Packet Delivery Rate (PDR) of the simulation results is observed and analyzed. PDR is an important performance 
metric to measure the performance and throughput of a network.

Simulation results showed that the Route Cache Timeout parameter significantly affects the throughput of the DSR protocol in mobile ad hoc networks. It's observed from the results that the effect of the Route Cache Timeout parameter on the PDR output of the DSR protocol is not same in high mobility and low mobility networks.

It can be concluded that before using the DSR protocol in a mobile ad hoc network, the mobility of the network should be properly analyzed and the Route Cache Timeout parameter should be set to a proper value according to the level of mobility.

\section{References}

[1] Perkins, C. E., \& Bhagwat, P. (1994). Highly dynamic destination-sequenced distance-vector routing (DSDV) for mobile computers. Proceedings of the Conference on Communications Architectures, Protocols and Applications: Vol. 24. Computer Communication Review (pp. 234-244). London, UK.

[2] Johnson, D., \& Maltz, D. (1996). Dynamic source routing in ad hoc wireless networks. Mobile Computing, 353, 153-181.

[3] Perkins, C. E., \& Royer, E. M. (1999). Ad-hoc on-demand distance vector routing. Proceedings of the Second IEEE Workshop on Mobile Computer Systems and Applications (pp. 90-100). New Orleans, Louisiana.

[4] Mohapatra, S., \& Kanungo, P. (2011). Performance analysis of AODV, DSR, OLSR and DSDV routing protocols using NS2 simulator. Proceedings of International Conference on Communication Technology and System Design: Volume 30, Procedia Engineering (pp. 69-76). Coimbatore, Tamil Nadu, India.

[5] Tuteja, A., Gujral, R., \& Thalia, S. (2010). Comparative performance analysis of DSDV, AODV and DSR routing protocols in MANET using NS2. Proceedings of International Conference on Advances in Computer Engineering: Advances in Computer Engineering (pp. 330-333). Bangalore, Karnataka, India.

[6] Tamilarasi, M., Das, S. K., \& Palanivelu, T. G. (2007). HART: Hop count based adaptive route cache timeout for efficient routing using DSR in MANETs. Proceedings of $13^{\text {th }}$ National Conference on Communications (pp. 203-207). Kanpur, India.

[7] Nikhil, I. P., \& Nael, B. A. (2002). Active route cache optimization for adhoc networks. Proceedings of $21^{\text {th }}$ Annual Joint Conference of the IEEE Computer and Communications Societies. New York, NY.

[8] Hu, Y. C., \& Johnson, D. (2000). Caching strategies in on-demand routing protocols for wireless ad hoc networks. Proceedings of the International Conference on Mobile Computing and Networks (pp. 231-242). Boston, Massachusetts.

[9] Kumar, A., Singh, P., Kumar, V., \& Tyagi, N. (2013). Performance analysis of AODV, CBRP, DSDV and DSR MANET routing protocol using NS2 simulation. International Journal of Computer Network \& Information Security, 5, 45-50.

[10] Abu-Salem, A. O., Samara, G., \& Alhmiedat, T. (2014). Performance analysis of dynamic source routing protocol. Journal of Emerging Trends in Computing and Information Sciences, 5, 97-100.

[11] Xiao, B., Zhuge, Q., Sha, Edwin, H. M., \& Chantrapornchai, C. (2002). Enhanced route maintenance for dynamic source routing in mobile ad hoc networks. In W. W. Smari, \& M. Guizani (Eds.). Proceedings of 15th ISCA International Conference on Parallel and Distributed Computing Systems. Louisville, Kentucky.

[12] Ishrat, Z., \& Singh, P. (2013). An enhanced DSR protocol using path ranking technique. International Journal of Engineering Research and Applications, 3, 1252-1256.

[13] Mathur, A. (2005). Performance Analysis and Enhancement of Dynamic Source Routing for Mobile Ad Hoc Networks. Honors thesis in computers science, University of Texas at San Antonio, College of Sciences, Division of Computer Science. 


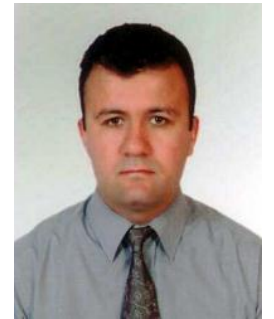

Barış Özyurt received his B.Sc. degree in computer engineering in 2000 from Middle East Technical University, Ankara, Turkey and a M.Sc. degree in computer engineering in 2003 from Middle East Technical University, Ankara, Turkey. He is currently attending the Ph.D. program in the Computer Science Department in Institute of Informatics at Gazi University, Ankara, Turkey. He has more than 10 years of experience as a software engineer in software industry. His research interests include mobile technologies, mobile ad hoc networks, web technologies and web mining.

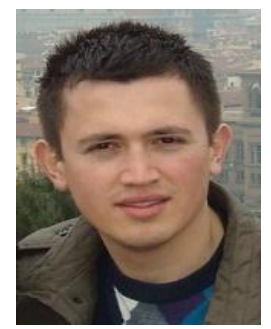

İbrahim Alper Doğru received his B.Sc degree in computer engineering in 2004 from Atlım University, Ankara, Turkey and a M.Sc. degree in computer engineering in 2007 from Gazi University, Ankara, Turkey and his Ph.D. degree in electronic-computer education in 2012 from the Gazi University, Ankara, Turkey. He is currently an Assist. Prof. Dr. at Gazi University, Department of Computer Engineering. His research interests include mobile network technologies, mobile ad hoc networks, mobile security, and cloud computing system.

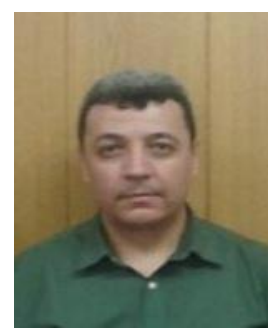

Muhammet Ali Akcayol received his B.Sc., M.Sc. and Ph.D. degrees from Gazi University, Institute of Science and Technology, Turkey. He is currently a Prof. Dr. at Gazi University, Department of Computer Engineering. His researches are focus on computer networks, wireless networks, mobile ad-hoc networks, and fuzzy logic. 